\title{
POLICIES FOR TEMPORAL PLACEMENT CONTROL OF VIDEO FRAMES IN B-ISDN NETWORKS
}

\author{
Duan-Shin Lee and Bhaskar Sengupta \\ C\&C Research Laboratory, NEC USA \\ 4 Independence Way \\ Princeton, New Jersey 08540
}

\begin{abstract}
In this paper, we propose a new method of traffic smoothing for frames of different video sources. We assume that each video source transmits frames at fixed intervals and that the network is free to decide the relative temporal spacing of video frames from different sources. This means that the time at which a given source begins to transmit its first frame is under the control of the network; however, after that, all frames from this source are transmitted at fixed intervals. We now address two questions. What are some reasonable policies for spacing the frames of video sources? What is the performance advantage of doing this? We propose three control policies (two heuristic and one based on an optimization problem via some Fourier analysis). These are compared against the case in which the video frames from different sources are randomly distributed (i.e., no control is exercised). We show that significant performance advantages accrue if one controls the temporal placement of the video frames from different sources.
\end{abstract}

Technical Subject Area: ATM systems and networks, Routing and control of congestion, admission, and flow.

correspondence should be sent to D.S. Lee. Tel:609-951-2456, Fax: 609-951-2499. 


\section{Introduction}

A recurring theme in traffic studies of B-ISDN has been to look for ways in which traffic can be smoothed, so that the network does not have to deal with extreme variation in bit-rates. This is usually achieved through flow control (through the leaky bucket and its variants $[8,15,16]$ ) and through congestion control (Distributed Source Control [11], Stop and Go queueing [3], Feedback control methods [2, 6, 10, 13, 18] and dynamic window methods [9]). In this paper, we address a problem which is somewhat similar in spirit to flow control methods, but very different in its specific aim and scope.

Consider a statistical multiplexer at the network ingress which is receiving traffic from various video sources. We assume that there are $K$ types of video calls. Type $k$ calls arrive according to a Poisson process at a rate $\lambda_{k}$ and that their call durations have a general distribution with mean $1 / \mu_{k}$. Each call, while in progress, generates a frame every $\tau$ ms $(\tau$ is 33 in most applications). The number of Mbits in a frame is modelled by using the video sources studied by Verbiest and Pinnoo [17]. We further assume that the output channel rate of the multiplexer is $C$ (e.g., $C=150 \mathrm{Mbps}$ ) and that the input channel rate for call type $k$ is $R_{k}$ (e.g., $R_{k}=150 \mathrm{Mbps}$ ). When a source of type $k$ transmits, it transmits a full frame as a burst at rate $R_{k}$, without any gaps. Thus if a particular frame has $X$ Mbits, the source of type $k$ transmits for $X \times 10^{3} / R_{k}$ ms and then remains silent for $\tau-X \times 10^{3} / R_{k} \mathrm{~ms}$. We assume that the stationary distribution of $X_{k}$ (the frame size) for type $k$ calls is given by $G_{k}(x)$, whose mean is $g_{k}$ (in Mbits). This implies that the mean bit-rate of a type $k$ call is $g_{k} \times 10^{3} / \tau$ Mbps. The multiplexer adopts the following admission control strategy. When a new video call arrives, we add the mean bit-rate of all currently active calls and the new call. The new call is admitted if this sum is less than or equal to $\sigma$ times $C$ (the output channel capacity of the multiplexer), otherwise it is not admitted. We now assume that marks are placed on the time axis at $0, \tau, 2 \tau, \cdots \mathrm{ms}$ and associated with each source $j$ is an offset $O_{j}$ $\left(0<O_{j}<\tau\right)$. This means that the frames corresponding to source $j$ begin to arrive at the multiplexer at times $O_{j}, \tau+O_{j}, 2 \tau+O_{j}, \cdots$. Let $k_{j}$ denote the type of call for source $j$. If 
the $i$ th frame from source $j$ is of size $X_{k_{j}}(i)$ (in Mbits), then the $i$ th frame from source $j$ is received at the multiplexer over the interval $\left[(i-1) \tau+O_{j},(i-1) \tau+O_{j}+X_{k_{j}}(i) \times 10^{3} / R_{k_{j}}\right]$. If no control is exercised on the video sources, then $O_{j}$ is assumed to be uniformly distributed between 0 and $\tau$. If this happens, then it is possible that the start times of the frames belonging to the currently active sources are temporally very close to one another, which may result in high input rate for some time (and consequently, lead to lost packets) and poor performance. To alleviate this situation, we need a mechanism which prevents the frames from different sources to be transmitted at or about the same time (i.e., prevent the frames from bunching). In other words, the start time of the frames from different sources should be evenly spread out over the interval of $\tau$ ms. If this could be achieved, we should be able to see less packet loss and better performance. We assume that this control is effected by the network by picking any value of $O_{j}$ it chooses. This choice is exercised only once per call, at the time of call admission. Once this number is determined for a particular call, it cannot be changed at a later time for the entire call duration (otherwise it may lead to synchronization problems at the destination). The problem is to find good ways of determining the $O_{j}$ for a newly arrived call, given that we know the offsets of the currently active calls. At this point, two questions arise. What are some reasonable ways of achieving this temporal placement control of video frames? What kind of performance gain can we expect to get as a result? We propose three methods for solving this problem. Two are heuristic and the last one involves a somewhat intricate optimization problem which we solve by using Fourier analysis.

This paper contains three more sections. In section 2, we outline the control policies. In section 3, we show the results and in section 4 , we present the conclusions.

\section{The Control Strategies}

Our two heuristics are quite simple and easy to understand. In the first heuristic, which we call the Midpoint method, we draw a circle whose circumference is $\tau$ and mark the offsets $O_{j}$ for the $j$ th active call on this circle. Note that we use the 12 o'clock position for an offset of 
zero and we measure the offsets in a clockwise direction from the 12 o'clock position. This means that any interval of the type $((i-1) \tau, i \tau]$ on the time axis is now represented by this circle, with the 12 o'clock position representing the point $i \tau$ for $i=1,2, \ldots$ If there are $n-1$ active calls, this divides the circle into $n-1$ intervals. If $O_{1} \leq O_{2} \leq \cdots \leq O_{n-1}$, then the intervals are $\left(O_{j}, O_{j+1}\right)$ for $j=1, \ldots, n-2$ and the last interval is from $O_{n-1}$ to $O_{1}$ in the clockwise direction. We now select the largest interval and choose the midpoint of this interval as the offset for the newly arrived call. The idea behind this method is that as calls depart, they do so in a random fashion. This randomness in the departures could leave the remaining calls somewhat bunched up. This method clearly tries to alleviate the bunching by choosing an offset which fills up the empty areas.

The second method is a just a little bit more difficult. It is called the Smallest Interval method. In this method, we draw a circle as before. For each source, we define an interval over which this source is expected to transmit. If there are $n$ active sources and $k_{j}$ denotes the type of source $j$, then this interval for source $j$ is defined as $\left[O_{j}, E_{j}\right]$, where $E_{j}=$ $\left\langle O_{j}+g_{k_{j}} \times 10^{3} / R_{k_{j}}\right\rangle$. Here, the angle brackets $(\langle\rangle)$ refer to modulo- $\tau$ arithmetic, so that all numbers remain between 0 and $\tau$. We now draw these $n$ intervals on the circle and shade them (see an example in Fig. 1). Clearly, with high probability, the shaded regions are areas on which the currently active sources are transmitting. Therefore, it would make sense for the newly arrived source to transmit on the unshaded areas. Our heuristic calls for first defining an interval of length $g_{k_{n}} \times 10^{3} / R_{k_{n}}$ for the $n$th call and try to fit it in the smallest unshaded interval. The fitting is done so that $O_{n}=E_{j}$ for some $j(j=1, \ldots, n-1)$. This means that the shaded regions from two sources will lie end-to-end on the circle. Note that as calls depart, they will leave unshaded gaps on the circle. It is possible that at some time, all of the unshaded intervals may be too small to fit this newly arrived call. If that happens, then we revert to the Midpoint method.

We now discuss a method based on an optimization problem. Again, assume that $n-1$ calls are currently in progress whose call types $\left(k_{j}\right)$, access line speeds $\left(R_{k_{j}}\right)$ and offsets $\left(O_{j}\right)$ are known (for $j=1, \ldots, n-1$ ). Assume further that call $n$ is of type $k_{n}$ and has an offset 
$O_{n}$, which is to be determined. From the knowledge of the parameters, we can come up with the expected bit-rate at time $t$, for $0<t<\tau$. Call this $P_{n}\left(t, O_{1}, \ldots, O_{n}\right)$. To clarify the meaning of $P_{n}(\cdot)$ further, we state that $\int_{0}^{x} P_{n}(t, \cdot) d t$ is the expected number of Mbits transmitted in the interval $(0, x)$. Our optimization problem attempts to choose a value of $O_{n}$ in such a way that the function $P_{n}(\cdot)$ has the greatest smoothness property. In other words, we seek to choose $O_{n}$ to minimize

$$
\int_{0}^{\tau} P_{n}^{2}\left(t, O_{1}, \ldots, O_{n}\right) d t-\left\{\int_{0}^{\tau} P_{n}\left(t, O_{1}, \ldots, O_{n}\right) d t\right\}^{2} .
$$

Clearly, minimizing this function is likely to reduce the extreme variation in bit-rates. We now give details of how this is accomplished.

Let $G_{k_{j}}(x)$ denote the distribution function of the number of Mbits generated in a frame by source $j$ and let $\bar{G}_{k_{j}}(x)$ denote its complementary function (i.e., $\bar{G}_{k_{j}}(x)=1-G_{k_{j}}(x)$ ). Let us concentrate on source $j$ for $1 \leq j \leq n$. We first assume that the offset $O_{j}$ is zero. The expected number of Mbits that arrive during $(t, t+d t)$ from source $j$ can be obtained by the following argument. Assume that the number of Mbits generated by this source in a particular frame is $x$. If $x>R_{k_{j}} t$, the number of Mbits that arrive in $(t, t+d t)$ is $R_{k_{j}} d t$. If $x \leq R_{k_{j}} t$, the number of Mbits that arrive in $(t, t+d t)$ is zero. Therefore, the expected bit-rate at time $t$ source $j$ is given by

$$
h_{j}(t)=R_{k_{j}} \bar{G}_{k_{j}}\left(R_{k_{j}} t\right) .
$$

Note that $h_{j}(t)$ is only defined for $0 \leq t<\tau$. Define the periodic version of $h_{j}(t)$ by $\tilde{h}_{j}(t)$, i.e.,

$$
\tilde{h}_{j}(t+k \tau)=h_{j}(t),
$$

for $k=0,1, \ldots$. If the offset $O_{j}$ is not zero, the expected bit-rate at time $t$ is given by

$$
f_{j}\left(t, O_{j}\right)=\tilde{h}_{j}\left(t-O_{j}\right) .
$$

From the definition of $P_{n}\left(t, O_{1}, O_{2}, \ldots, O_{n}\right)$, we have

$$
P_{n}\left(t, O_{1}, O_{2}, \ldots, O_{n}\right)=\sum_{j=1}^{n} f_{j}\left(t, O_{j}\right)=\sum_{j=1}^{n} \tilde{h}_{j}\left(t-O_{j}\right) .
$$


We need to find the optimal $O_{n}$ that minimizes the variance of $P_{n}\left(t, O_{1}, O_{2}, \ldots, O_{n}\right)$, i.e., minimizes (1). But $\int_{0}^{\tau} P_{n}\left(t, O_{1}, O_{2}, \ldots, O_{n}\right) d t$ is the expected number of Mbits transmitted in $(0, \tau]$ and equals $\sum_{j=1}^{n} g_{k_{j}} \times 10^{3} / \tau$. So the problem is equivalent to

$$
\min _{0 \leq O_{n}<\tau} \int_{0}^{\tau} P_{n}^{2}\left(t, O_{1}, O_{2}, \ldots, O_{n}\right) d t
$$

To carry out the minimization in Eq.(3), we first assume that the number of Mbits generated by a video source of type $k$ in a frame can be represented by a truncated and shifted second-degree Erlang random variable $X_{k}$. This is a rich two parameter family of distributions (the parameters are $\alpha_{k}$ and $s_{k}$ ) which should meet most modeling needs. The density function of this random variable is

$$
P\left(x<X_{k} \leq x+d x\right)= \begin{cases}B \alpha_{k}^{2}\left(x-s_{k}\right) \exp \left(-\alpha_{k}\left(x-s_{k}\right)\right) d x & \text { if } s_{k} \leq x<R_{k} \tau \\ 0 & \text { otherwise, }\end{cases}
$$

where $B$ is a suitable normalization constant which makes the density integrate to one. Since $\tau R_{k}$ (the number of Mbits that can be transmitted in time $\tau$ ) is much greater than the expected value of $X_{k}$, the normalization constant $B$ is actually very close to one in Eq.(4). In the numerical results (to follow), we allow values of $K=1,2$. For source type 1, we chose $\alpha_{1}=9.411$ (1/Mbits) and $s_{1}=3.467$ (Mbits). With these choices, the mean and standard deviation of $X_{1}$ turn out to be 0.559 Mbits and 0.15 Mbits. This is a good approximation because Verbiest and Pinnoo [17] report a mean and standard deviation of 0.56 and 0.143 Mbits respectively. We further assume that the type 2 sources are a scaled version of type 1 sources by a factor of 0.5 , then $\alpha_{2}=\alpha_{1} / 0.5$ and $s_{2}=0.5 s_{1}$. Note that this scaling preserves all other characteristics (such as autocorrelations) of the sources of type 1.

The complementary distribution of $X_{k}(k=1, \ldots, K)$ is

$$
\bar{G}_{k}(x)= \begin{cases}B \exp \left(-\alpha_{k}\left(x-s_{k}\right)\right)\left(\alpha_{k}\left(x-s_{k}\right)+1\right), & \text { if } s_{k}<x<\tau R_{k} \\ 1, & \text { if } 0 \leq x \leq s_{k}\end{cases}
$$

Therefore,

$$
h_{k}(t)=R_{k} \bar{G}_{k}\left(R_{k} t\right)
$$




$$
= \begin{cases}R_{k}, & \text { if } 0 \leq t<s / R_{k} \\ R_{k} B \exp \left(-\alpha_{k}\left(t-s_{k}\right)\right)\left(\alpha_{k}\left(t-s_{k}\right)+1\right), & \text { if } s_{k} / R_{k}<t<\tau .\end{cases}
$$

The Fourier coefficients of $\tilde{h}_{k}(t)$ are now given by

$$
\begin{aligned}
c_{m}(k)= & \frac{1}{\tau} \int_{0}^{\tau} \tilde{h}_{k}(t) \exp \left(-\frac{2 \pi m i t}{\tau}\right) d t \\
= & \frac{R_{k}}{2 \pi m i}\left(1-\exp \left(-\frac{2 \pi m i s_{k}}{R_{k} \tau}\right)\right)+\frac{2 \exp \left(-\frac{2 \pi m i s_{k}}{R_{k} \tau}\right)\left(\pi m i+\alpha_{k} R_{k} \tau\right) R_{k} B}{\left(2 \pi m i+\alpha_{k} R_{k} \tau\right)^{2}}, \\
& \quad \text { for }-\infty<m<\infty, k=0,1, \ldots, n .
\end{aligned}
$$

Note that a different assumption on $\bar{G}_{k}(x)$ in $(5)$ would lead to a different result for $c_{m}(k)$. In all other respects, the methodology remains the same. Let $\left\{\phi_{m}\right\}$ denote the Fourier coefficients of $P_{n}\left(t, O_{1}, \ldots, O_{n}\right)$. Then, from (2),

$$
\phi_{m}=\sum_{j=1}^{n} c_{m}\left(k_{j}\right) \exp \left(-\frac{2 \pi m i O_{j}}{\tau}\right) .
$$

Using Parseval's theorem ([1]), we have

$$
\int_{0}^{\tau} P_{n}^{2}\left(t, O_{1}, \ldots, O_{n}\right) d t=\sum_{m=-\infty}^{\infty}\left|\phi_{m}\right|^{2}
$$

To carry out the minimization in (3), we allow $L$ possible choices of $O_{n}$ on a grid, so that $O_{n}=\tau(\ell-1) / L$ for $\ell=1, \ldots, L$. Then, we choose that value of $\ell$ which minimizes $(6)$.

Since $c_{m}(k)$ is of the order $m^{-1}$, Eq.(6) has the same rate of convergence as the series $\sum m^{-2}$. While this may be satisfactory in some applications, it is possible to speed up this rate of convergence further. We now show how to do this. Define

$$
\begin{array}{rlrl}
J_{k} & =h_{k}(0+)-h_{k}(\tau-) \text { for } & k=1, \ldots, K, \\
\xi(t+j \tau) & =t, & & 0 \leq t<\tau, j=0,1, \ldots, n
\end{array}
$$

and

$$
P_{n}^{*}\left(t, O_{1}, \ldots, O_{n}\right)=P_{n}\left(t, O_{1}, \ldots, O_{n}\right)+\sum_{j=1}^{n} \frac{J_{k_{j}}}{\tau} \xi\left(t-O_{j}\right)
$$


Note that $P_{n}\left(t, O_{1}, \ldots, O_{n}\right)$ has $n$ discontinuities at $t=O_{1}, \ldots, O_{n}$, while $P_{n}^{*}\left(t, O_{1}, \ldots, O_{n}\right)$ is continuous. Let $\left\{d_{m}\right\}$ denote the Fourier coefficients of $\sum_{j=1}^{n} \frac{J_{k_{j}}}{\tau} \xi\left(t-O_{j}\right)$. Then

$$
d_{m}= \begin{cases}\sum_{j=1}^{n} \frac{J_{k_{j}}}{2}, & \text { if } j=0 \\ \sum_{j=1}^{n} \frac{-J_{k_{j}}}{2 \pi m i} \exp \left(-\frac{2 \pi m i O_{j}}{\tau}\right), & \text { if } j \neq 0 .\end{cases}
$$

Let $\left\{\phi_{m}^{*}\right\}$ denote the Fourier coefficients of $P_{n}^{*}\left(t, O_{1}, \ldots, O_{n}\right)$. From Eq.(7),

$$
\begin{aligned}
\left|\phi_{m}\right|^{2} & =\left|\phi_{m}^{*}-d_{m}\right|^{2} \\
& =\left|\phi_{m}^{*}\right|^{2}+\left|d_{m}\right|^{2}-2 \operatorname{Re}\left(\phi_{m} \bar{d}_{m}\right),
\end{aligned}
$$

where $\bar{d}_{m}$ is the complex conjugate of $d_{m}$. Substitute Eq.(9) in Eq.(6) to get

$$
\sum_{m=-\infty}^{\infty}\left|\phi_{m}\right|^{2}=\sum_{\substack{m=-\infty \\ m \neq 0}}^{\infty}\left|\phi_{m}^{*}\right|^{2}+\sum_{\substack{m=-\infty \\ m \neq 0}}^{\infty}\left|d_{m}\right|^{2}-2 \sum_{\substack{m=-\infty \\ m \neq 0}}^{\infty} \operatorname{Re}\left(\phi_{m}^{*} \bar{d}_{m}\right)+\left|\phi_{0}^{*}-d_{0}\right|^{2},
$$

The second term on the right hand side of Eq.(10) can be simplified as follows:

$$
\begin{aligned}
\left|d_{m}\right|^{2} & =\frac{1}{4 \pi^{2} m^{2}}\left[\left(\sum_{j=1}^{n} J_{k_{j}} \cos \frac{2 \pi m O_{j}}{\tau}\right)^{2}+\left(\sum_{j=1}^{n} J_{k_{j}} \sin \frac{2 \pi m O_{j}}{\tau}\right)^{2}\right] \\
& =\frac{1}{4 \pi^{2} m^{2}}\left[\sum_{j=1}^{n} J_{k_{j}}^{2}+\sum_{\substack { \ell=1 \\
\begin{subarray}{c}{j=1 \\
j \neq \ell{ \ell = 1 \\
\begin{subarray} { c } { j = 1 \\
j \neq \ell } }\end{subarray}}^{n} J_{k_{\ell}} J_{k_{j}} \cos \frac{2 \pi m\left|O_{\ell}-O_{j}\right|}{\tau}\right] .
\end{aligned}
$$

Let $B_{2}(x)=x^{2}-x+6^{-1}$ denote the Bernoulli polynomial of second degree and let $\tilde{B}_{2}(x)$ be the periodic version of $B_{2}(x)$, i.e., $\tilde{B}_{2}(x+m \tau)=B_{2}(x)$ for $m=0,1, \ldots$ and $0 \leq x<\tau$. From the well known identities ([5])

$$
\tilde{B}_{2}(x)=\sum_{m=1}^{\infty} \frac{\cos 2 \pi m x}{\pi^{2} m^{2}} \text { and } \sum_{m=1}^{\infty} \frac{1}{m^{2}}=\pi^{2} / 6
$$

Eq.(10) becomes

$$
\begin{gathered}
\sum_{m=-\infty}^{\infty}\left|\phi_{m}\right|^{2}=\left|\phi_{0}^{*}-d_{0}\right|^{2}+\sum_{j=1}^{n} \frac{J_{k_{j}}^{2}}{12}+\sum_{\ell=1}^{n} \sum_{\substack{j=1 \\
j \neq \ell}}^{n} \frac{J_{k_{\ell}} J_{k_{j}}}{2} B_{2}\left(\frac{\left|O_{\ell}-O_{j}\right|}{\tau}\right)+ \\
2 \sum_{m=1}^{\infty}\left|\phi_{m}^{*}\right|^{2}-4 \sum_{m=1}^{\infty} \operatorname{Re}\left(\phi_{m}^{*} \bar{d}_{m}\right) .
\end{gathered}
$$


Since the derivative of $P_{n}^{*}\left(t, O_{1}, \ldots, O_{n}\right)$ with respect to $t$ has bounded variation [14], $\phi_{m}^{*}$ is of order $m^{-2}([4,1])$. Hence, Eq.(11) has the same convergence rate as the series $\sum m^{-3}$ which is much better than that of (6). We state that it is possible to speed up the convergence rate even more by adding a quadratic (or higher order) term to Eq.(7), which guarantees the existence of the second (or higher order) derivative of $P_{n}\left(t, O_{1}, \ldots, O_{n}\right)$ with respect to $t$

([4]). However, this is possible at the cost of considerable extra work. Further, we were able to obtain very satisfactory results with the method presented above.

\section{$3 \quad$ Numerical Results}

We study the effect of the three policies by simulation. We use a fluid-flow model so as to keep the run time for the simulations within reasonable limits [7]. The number of Mbits in a frame $\left(X_{k}\right)$ is modelled by using the sum of two autoregressive processes and a Markov chain (see Ramamurthy and Sengupta [12]). This model matches the bit-rate histogram and the autocorrelation function for video sources studied by Verbiest and Pinnoo [17]. The mean bit-rate of these sources is $16.8 \mathrm{Mbps}$ (which corresponds to $0.56 \mathrm{Mbits}$ per frame). If $K=2$, then we consider two types of sources, one of which is high bit-rate (same as the one in [17] and [12]) and the other whose bit-rate is scaled by a factor of 0.5 (i.e., half the bit-rate, but otherwise preserving all other characteristics of the sources in [17] and [12]). The sources arrive according to a Poisson process and may or may not be admitted to the multiplexer according to the admission control strategy given in section 1. Thus, the number of active sources in our simulation keeps on changing with time. The call holding time is assumed to be distributed uniformly between 5400 and 9000 frames. We assume that a layered coding $[7,12]$ scheme has been employed and therefore, we are mainly interested in the loss rate of the high priority cells. The buffer threshold to discard low priority cells is 50 (in cells) and the total buffer size of the multiplexer is 300 cells. In order to faithfully compare the three policies, we simulate four systems (three control policies and the system without control) subject to the same arrival processes and holding times of video sources. Specifically, we 
generate one sequence of call arrivals and holding times and feed this sequence to all four systems. This means that all four systems receive the same number of Mbits per frame from all accepted calls. In all the results reported here, $C=150 \mathrm{Mbps}, R_{k}=150 \mathrm{Mbps}$ for $k=1,2, \sigma=0.95$ and $L=33$.

In the first set of comparisons (see Fig. 2), we assume that $K=1$, i.e., the sources are homogeneous. The sources arrive according to a Poisson process of rate $0.03(1 / \mathrm{sec})$. we compute the loss rate of the high priority cells conditioned on the fact that $n$ sources are active. This conditional loss probability is plotted against $n$. It is clear from Fig. 2 that both the Fourier analysis method and the midpoint method outperform the smallest interval method. Further, all three are much better than having no control at all. It is not difficult to understand the qualitative aspect of this result. The smallest interval method places the shaded arcs end-to-end on the circle. This means that we are avoiding severe bunching of the offsets, i.e., the offsets are not too close to each other. However, if there are only two or three sources which have just started transmission, then their shaded regions will lie close to one another in one part of the circle. This is to be contrasted with the midpoint method, in which if two or three sources have just started transmission, their offsets will be very far apart from each other. This explains why this method is better than no control and why the midpoint method is so much better than the smallest interval method. The Fourier analysis method in this case performs about the same as the midpoint method. The reason why the two graphs do not coincide is that the criterion of optimality (greatest smoothness in expected bit-rate) is different from the criterion of loss rate (which is what is shown in Fig. 2). Moreover, the fact that the Fourier analysis method chooses a point on a discrete grid makes it non-optimal.

For the case when $K=2$, which we call the heterogeneous case, the results are shown in Figs. 3 and 4. The sources of both types arrive according to Poisson processes of rate $0.015(1 / \mathrm{sec})$. Again, the results are obtained by a single simulation run in which the loss rate of high priority cells of both types of calls conditioned on $n_{1}$ type 1 calls and $n_{2}$ type 2 calls being active. Then, Fig. 3 is plotted with $n_{1}=3$ with $n_{2}$ as the $\mathrm{x}$-axis and Fig. 
4 is the corresponding graph with $n_{1}=5$. In both of these graphs, it is obvious that having any control method is far better than having no control. But this time, note that the Fourier analysis method far outperforms the two heuristics. This is intuitive, since the two heuristics were not designed specifically for heterogeneous traffic. Imagine that $n_{1}=n_{2}=1$. The midpoint method would try to place one offset at 0 and the other at $\tau / 2$ without taking into consideration the fact that the two sources are very different in size. The Fourier analysis method, being analytical, takes into account these factors automatically! It places the offset of the type 1 source at 0 and that of the type 2 source at about $2 \tau / 3$, since a type 1 source has twice the bit-rate of a type 2 source. Also observe that when $n_{2}$ becomes large, the two heuristics perform about equally well. This is also to be expected. When the circle is well filled with shaded regions, two things happen. Either the shortest interval method reverts to the Midpoint method or the shaded arcs lie reasonably close to one another with a small amount of gap. In either case, its behavior is not expected to be too different from that of the Midpoint method. The low priority cell loss rate is shown in Fig. 5 for $n_{1}=3$. The complementary distribution of queue length (unconditional) during the entire simulation is shown in Fig. 6. Again the Fourier analysis method provides the best performance for the low priority cell loss rate and the queue length distribution.

In addition to the loss probability, we have used another measure to compare the three policies. This measure is the smoothness index $(\eta)$, which is the square root of the quantity in Eq. (1). We take the square root of the quantity in Eq. (1) because it has the more natural units of Mbits. The computation of the smoothness index was done by two simulation runs, one for the homogeneous case and the other for the heterogeneous case. For the homogeneous and the heterogeneous cases, we show the (unconditional) value of the smoothness index in Table 1 for the problem in which no control is exercised as well as the three control policies. As expected, the smoothness index is lowest for the Fourier analysis method, followed very closely by the Midpoint method. The difference between these two and the Smallest interval method as well as the no control case is more. Even by this criterion, the Fourier analysis method and the Midpoint methods give much better results. Note that the difference between 


\begin{tabular}{|c|c|c|c|c|}
\hline & no control & smallest interval & midpoint & Fourier analysis \\
\hline homogeneous & 26.2 & 22.3 & 21.4 & 21.5 \\
\hline heterogeneous & 23.5 & 20.3 & 19.9 & 19.5 \\
\hline
\end{tabular}

Table 1: Smoothness index (in the unit of Mbits) for the homogeneous case and the heterogeneous case.

the Fourier analysis method and the Midpoint method is quite small. This is to be contrasted with the conditional loss probabilities reported for the heterogeneous case, in which the difference between these two methods was much higher. The reason this difference occurs is because for bursty traffic, it is possible to have a difference in measures such as loss probabilities while more robust measures like mean and variance of queue length show very little difference. Our smoothness measure is (in some sense) measuring the variation in the input rate. While this measure correctly preserves the relative ranking of the different control methods, it does not give the complete picture about the loss probabilities.

\section{Conclusions}

We have examined three different policies for temporal placement control of video frames. Any reasonable form of control is much better than doing no control. Of the three methods, the Midpoint method is very simple and performs as well as the Fourier analysis method for homogeneous sources. However, for heterogeneous sources, the Fourier analysis method is the best by far.

\section{References}

[1] N. K. Bary, “A treatise on trigonometric series", Vol. 1, Pergamon Press, New York.

[2] J. Bolot and A. U. Shankar, "Dynamical behavior of rate-based flow control mechanism", Computer Communication Review, April 1990. 
[3] S. J. Golestani, "A Stop-and-Go Queueing Framework for Congestion Management," Sigcomm'90, Philadelphia, PA, September 1990.

[4] D. L. Jagerman, "Mathcalc", unpublished manuscript.

[5] K. Knopp, “Theory and application of infinite series," Blackie \& Son Limited, London.

[6] K. Ko, P. P. Mishra and S. K. Tripathi, "Predictive Congestion Control in High Speed Wide Area Networks," Proc. Second IFIP WG6.1/W664 International Workshop on Protocols for High Speed Networks, California, November, 1990.

[7] D-S. Lee, B. Melamed, A. R. Reibman and B. Sengupta, "TES modeling for analysis of a video multiplexer," Performance Evaluation, Vol. 16, pp. 21-34, 1992.

[8] K. K. Leung, B. Sengupta and R. W. Yeung, "A Credit Manager for Traffic Regulation of High Speed Networks: A Queueing Analysis", IEEE Trans. in Networking, Vol. 1 (1993), No. 2, pp. 236-245.

[9] D. Mitra, "Dynamic Adaptive Windows for High Speed Data Networks: Theory and Simulation," Proc. ACM SIGCOMM '90, 1990, pp. 30-37.

[10] A. Mukherjee and J. C. Strikwerda, "Analysis of Dynamic Congestion Control Protocols - A Fokker-Planck Approximation", ACM SIGCOMM'91, Zurich, Switzerland.

[11] G. Ramamurthy and R. Dighe, "Distributed Source Control: A Network Access Control for Integrated Broadband Packet Networks," IEEE Journal on Selected Areas in Communication, September, 1991, Vol. 9, No. 7, pp. 990-1002.

[12] G. Ramamurthy and B. Sengupta, "Modeling and Analysis of a Variable Bit Rate Video Multiplexer", Infocom'92, pp. 817-827, Florence, Italy, 1992.

[13] G. Ramamurthy and B. Sengupta, "A Predictive Hop-by-Hop Congestion Control Policy for High Speed Networks," Infocom'93, pp. 1033-1041, San Francisco, California, 1993.

[14] H. L. Royden, "Real Analysis," second ed., The Macmillan Company, New York. 
[15] K. Sohraby and M. Sidi, "On the Performance of Bursty and Correlated Sources Subject to Leaky Bucket Rate-based Access Control Schemes," Infocom '90, pp. 426-434.

[16] J.S. Turner, "New directions in communications (or which way to the information age?)", IEEE Communications Magazine, Oct. 1986.

[17] W. Verbiest and L. Pinnoo, "A variable rate video codec for asynchronous transfer mode networks," IEEE J. on Selected Areas in Commun., Vol. 7, No. 5, pp. 761-770, June 1989.

[18] Y. T. Wang and B. Sengupta, "Performance of a Feedback Congestion Central Policy Under Non-negligible Propagation Delay," Proc. of ACM SIGCOMM '91, September, 1991, pp. 149-157. 
Figure 1: Smallest interval method. 


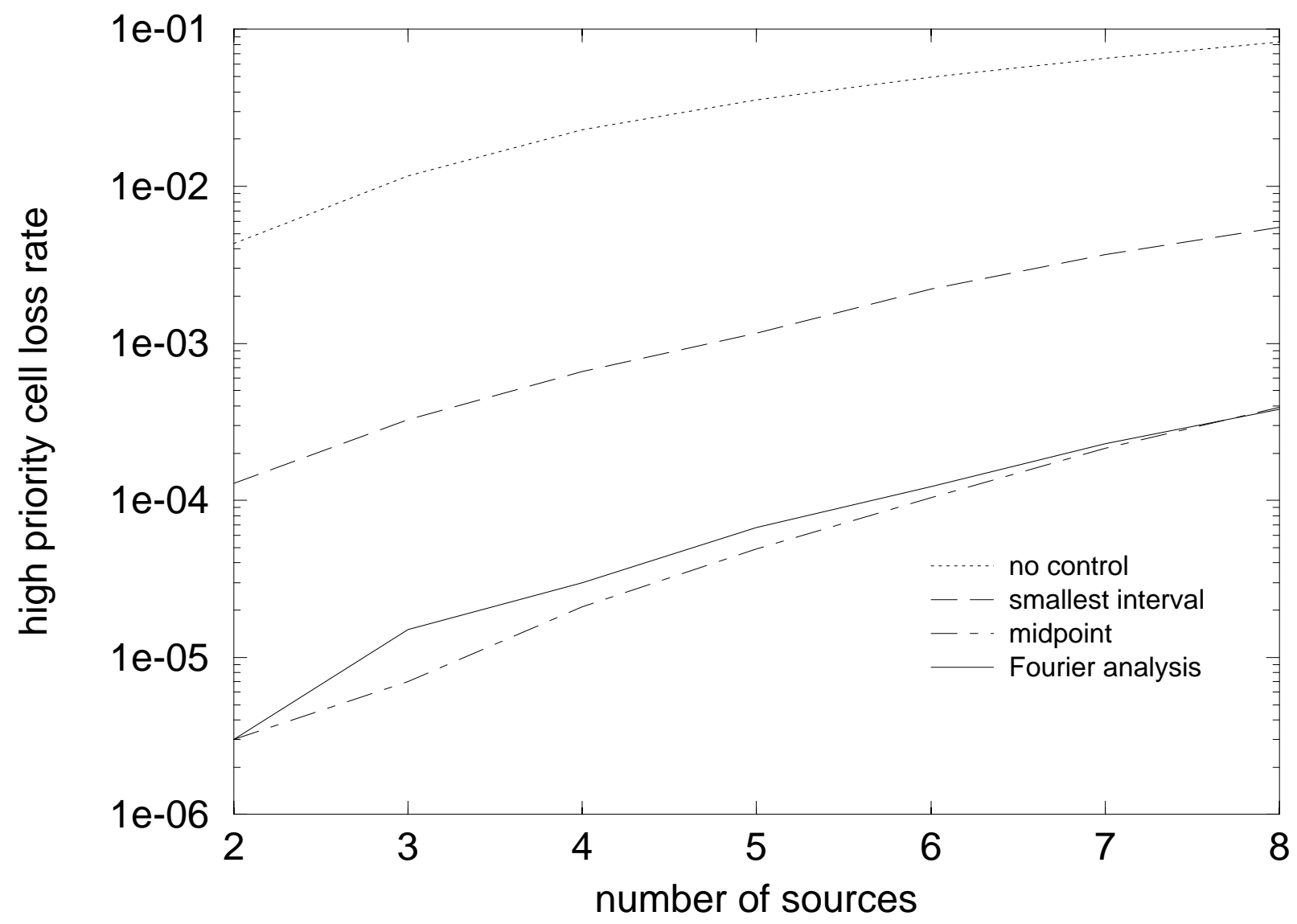

Figure 2: High priority cell loss rate in the case of homogeneous video sources. 


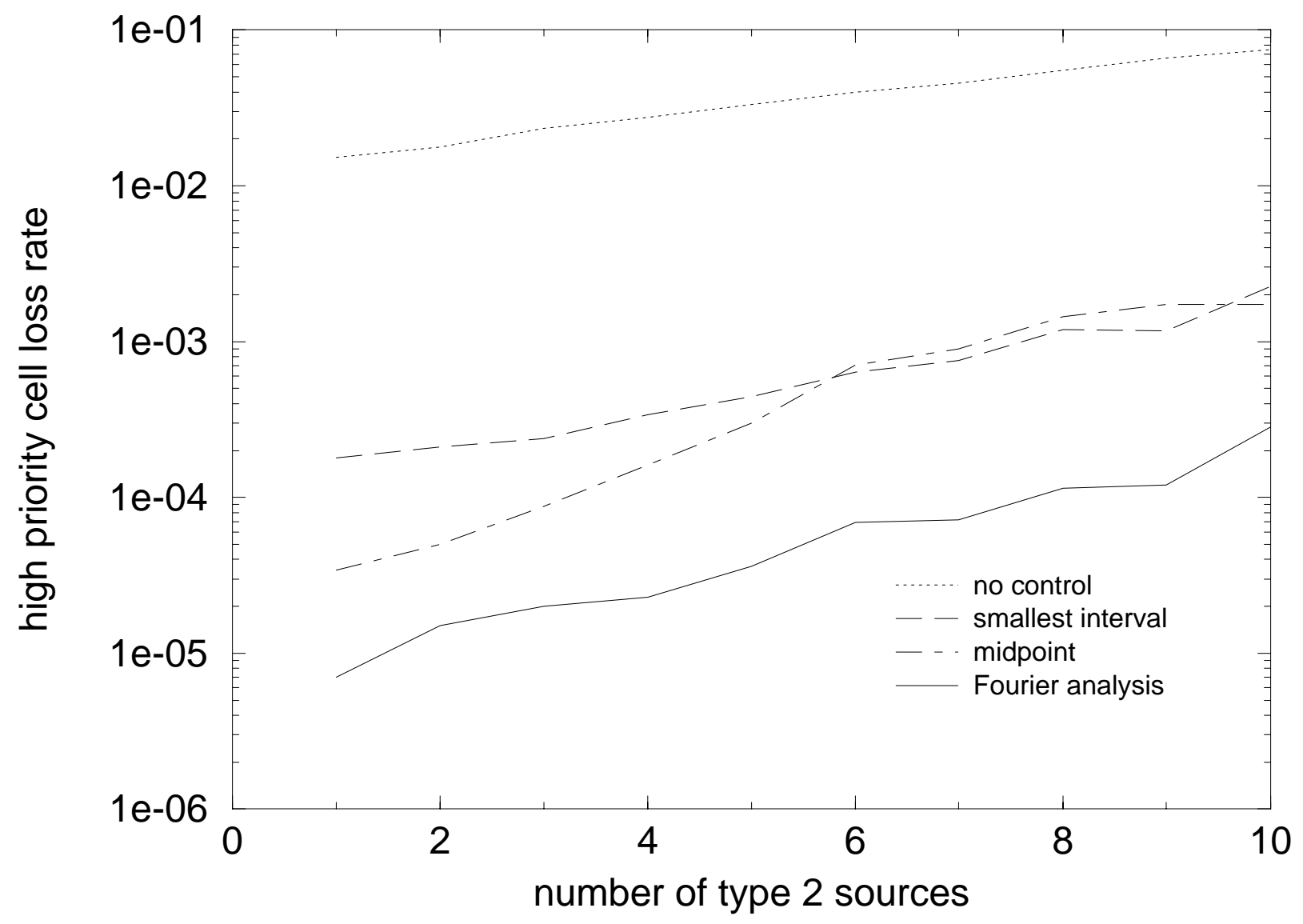

Figure 3: High priority cell loss rate in the case of heterogeneous video sources. Number of type 1 sources equals to 3 . 


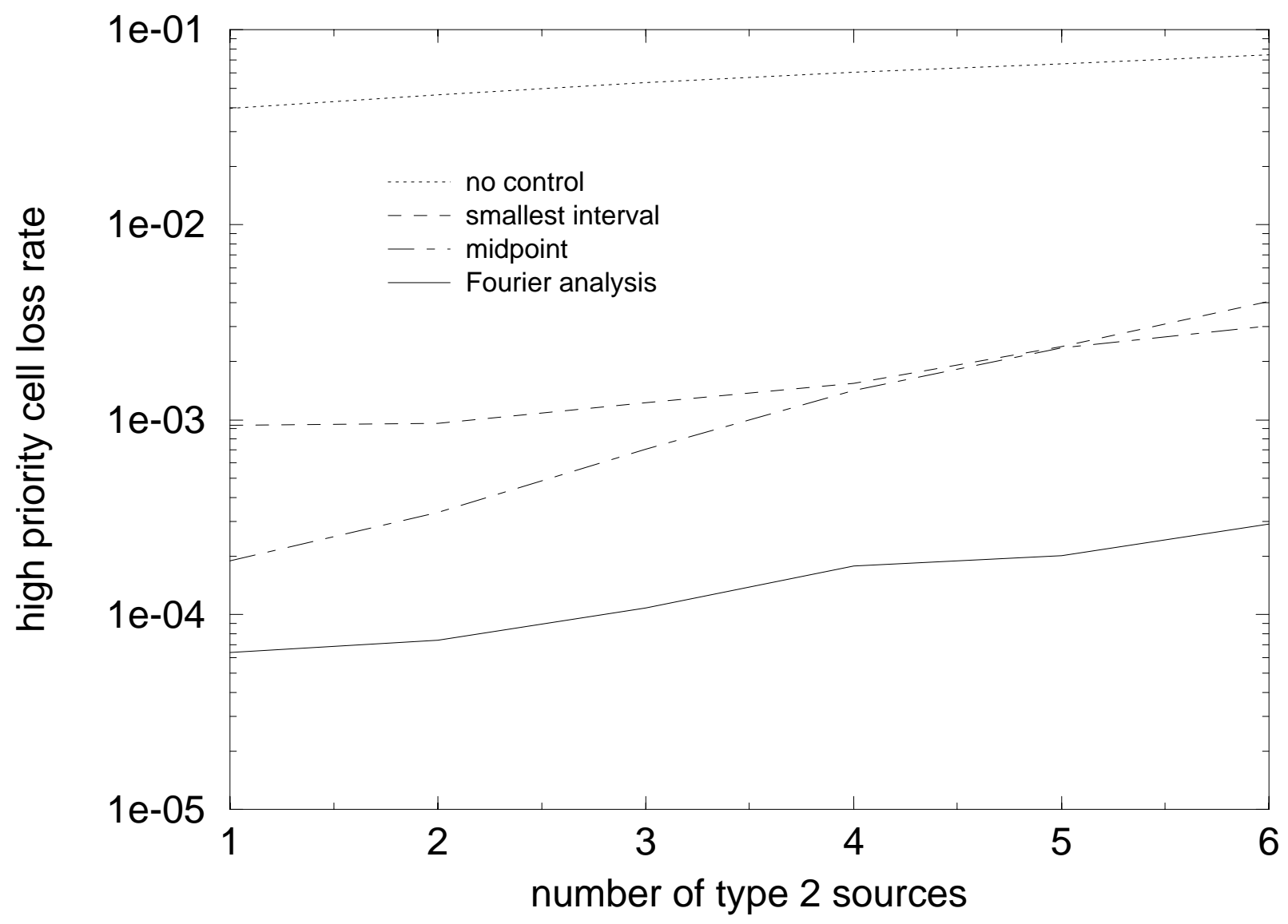

Figure 4: High priority cell loss rate in the case of heterogeneous video sources. Number of type 1 sources equals to 5 . 


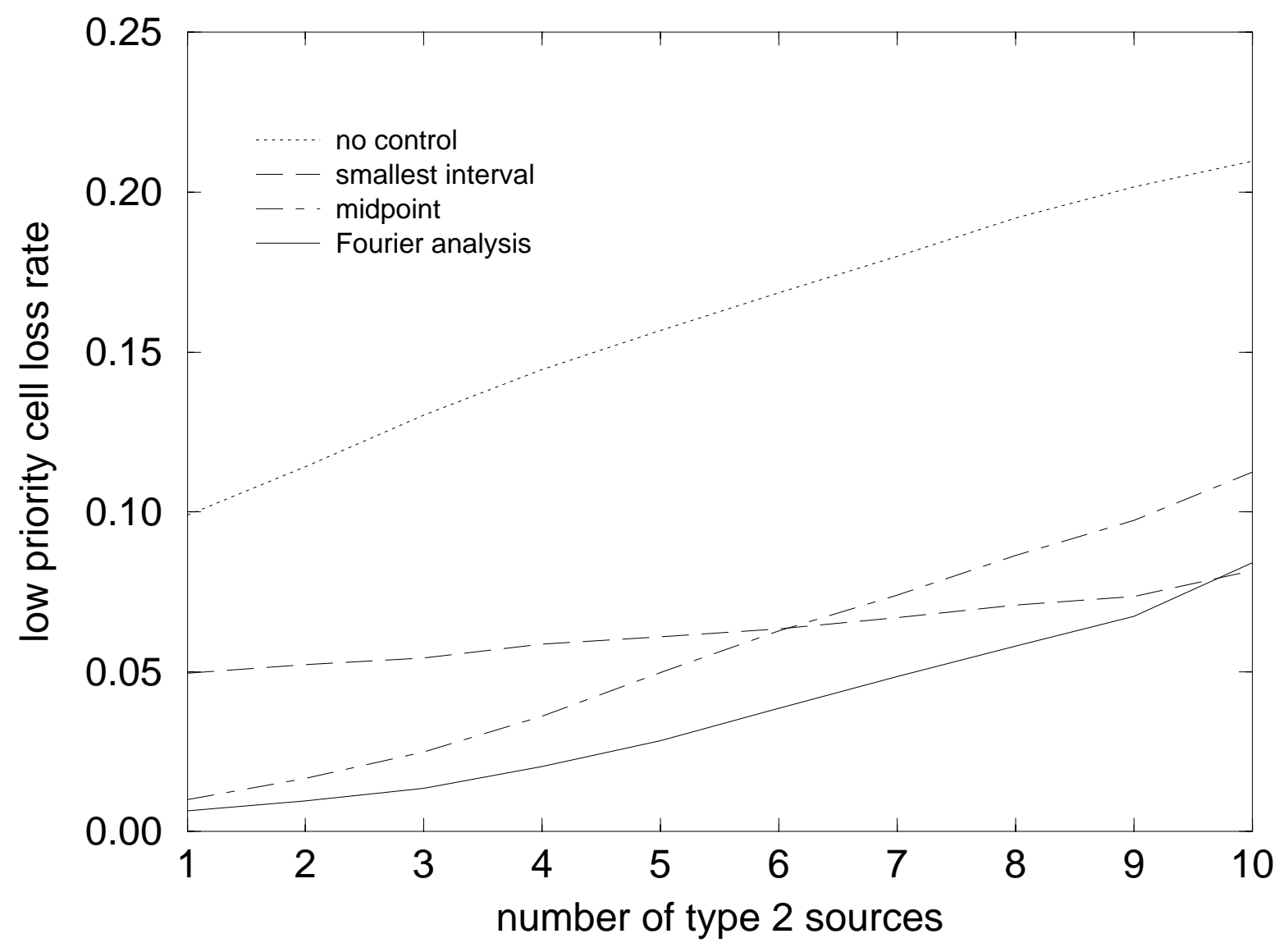

Figure 5: Low priority cell loss rate in the case of heterogeneous video sources. Number of type 1 sources equals to 3 . 


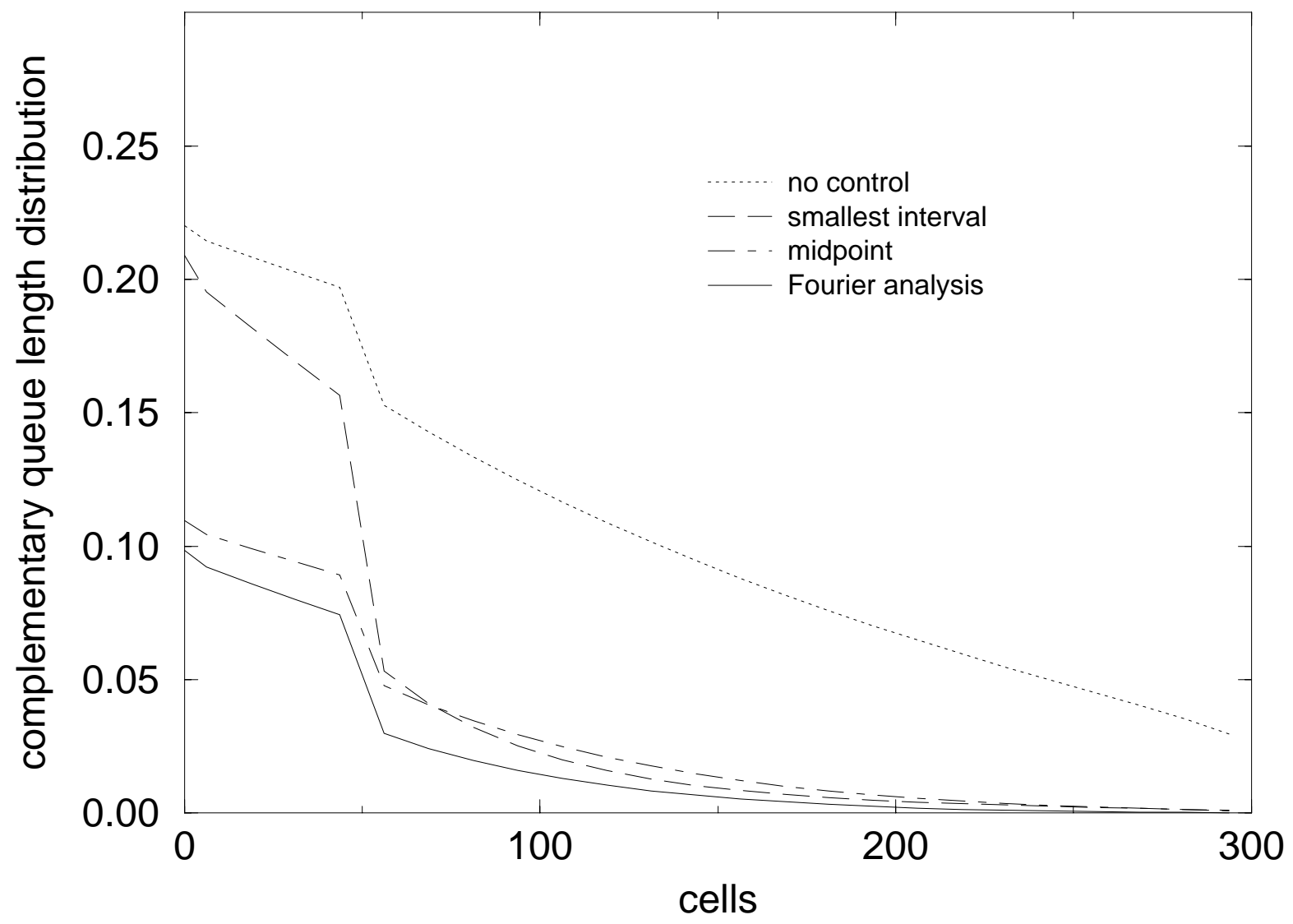

Figure 6: Complementary queue length distribution in the heterogeneous case. 